\title{
OpenNotes: Transparenz steigert Vertrauen und Patientensicherheit
}

\author{
Erika Ziltener ${ }^{\mathrm{a}}$, Andréa Belliger ${ }^{\mathrm{b}}$, Karin Fattinger ${ }^{\mathrm{c}}$ \\ a lic. phil., Historikerin und diplomierte Pflegefachfrau, Geschäftsleiterin der Patientenstelle Zürich und Präsidentin des Dachverbandes der Schweizerischen \\ Patientenstellen; ${ }^{b}$ Prof. Dr. theol., Digital-Health-Expertin, Prorektorin Pädagogische Hochschule Luzern und Leiterin Institut für Kommunikation \& Führung \\ IKF Luzern; ' Prof. Dr. med., Fachärztin für Allgemeine Innere Medizin, Vorstandsmitglied der Patientenstelle Zürich und des Dachverbandes Schweizerischer \\ Patientenstellen
}

Ärzte, die ihre ärztlichen Verlaufseinträge für ihre Patienten öffnen, stärken die Arzt-Patienten-Beziehung und das Vertrauen. Die Patienten übernehmen vermehrt Verantwortung für sich selbst und ihre Behandlung. Die Patientensicherheit verbessert sich. Eine partizipative Entscheidungsfindung wird gefördert. All dies kann erreicht werden, ohne dass die Arbeitslast der Ärzte steigt.

\begin{abstract}
Die Partizipative Medizin (Partizipative Entscheidungsfindung, Shared Decision Making) beinhaltet, dass bei der Entscheidungsfindung neben der wissenschaftlichen Evidenz die Werte und Präferenzen des Patienten gleichwertig einbezogen werden [1]. Arzt und Patient entscheiden sich gemeinsam auf Augenhöhe für die Behandlung, die am besten den Bedürfnissen des $\mathrm{Pa}-$ tienten entspricht.
\end{abstract}

\section{Résumé}

La médecine participative tient non seulement compte des faits scientifiques, mais attache une égale importance aux valeurs et préférences des patients. Les patients émancipés veulent avoir accès à leur documentation médicale. Le mouvement international OpenNotes vise à rendre plus transparente la coopération entre les patients et les professionnels de santé. Les patients peuvent consulter les notes des professionnels, d'où le nom d'OpenNotes (notes consultables par les patients).

Une étude publiée en 2012 sur la mise en œuvre d'OpenNotes auprès de plus de 10000 patients et 105 médecins de famille fait état de résultats unanimement positifs. Les patients apprécient vivement le partage des dossiers médicaux. Pour les médecins, la charge de travail est la même et les processus sont à peine différents. Avec les notes en libre accès, les patients assument davantage la responsabilité de leur santé et de leur traitement. OpenNotes améliore la relation et la confiance entre le médecin et les patients, ainsi que la satisfaction de ces derniers. D'autres études confirment ces résultats positifs.

En 2019, plus de 38 millions de patients profitent de ce système aux Etats-Unis. A cela s'ajoutent les patients de 19 pays supplémentaires. L'utilité d'OpenNotes est largement démontrée. Le système contribue à une médecine participative, axée sur le patient.

Etant donné les expériences internationales positives et la tendance à la médecine participative, I'introduction dans les meilleurs délais d'OpenNotes en Suisse aurait du sens.
Heute sind umfassende Informationen zu Gesundheitsthemen bzw. Krankheiten für alle transparent und frei verfügbar. Wobei es zu beachten gilt, dass im Internet Informationen unterschiedlichster Qualität vorliegen und wir alle, kranke wie gesunde Personen, Laien wie Fachleute, lernen müssen, mit Quellen unterschiedlicher Qualität adäquat umzugehen.

Im Rahmen des Kulturwandels zu einer Partizipativen Medizin wünschen sich viele Patienten, ihre Patientendokumentation einzusehen. In den USA haben Patienten seit 1996 das Recht, Kopien zu erhalten und Ergänzungen zu verlangen [2]. In der Schweiz verfügen Patienten auch über diese Rechte [3].

\section{Internationale Bewegung}

OpenNotes ist eine internationale Bewegung, die von Patienten sowie Fachleuten in den USA ins Leben gerufen wurde und heute in vielen Ländern präsent ist. Ihr Ziel ist, die Zusammenarbeit zwischen Patienten und Fachleuten transparenter zu gestalten. Die Patienten werden aufgefordert, die Notizen (englisch Notes) ihrer Ärzte und anderer an der Behandlung beteiligter Fachleute zu lesen - daher der Begriff OpenNotes, für den Patienten offengelegte Notizen.

1973 wird erstmals gefordert, Patienten eine Kopie ihrer Dokumentation auszuhändigen [4]. Die Ziele sind Qualität, Kontinuität und weniger Bürokratie. Die Patientenbewegung fordert Patienten auf, sich aktiv bei ihrer Behandlung einzubringen.

1996 verlangt der Health Insurance Portability and Accountability Act (HIPPA) für die USA nationale Standards für die Elektronische Gesundheitsvorsorge. Die Patienten erhalten das Recht, ihre Patientendokumen- 
tation einzusehen, zu kontrollieren und eine Kopie zu erhalten [2]. Das neue Gesetz, das in anderen Ländern nachvollzogen wird, ebnet den Weg für die OpenNotesBewegung.

Seit 1998 stellen Gesundheitsinstitutionen in den USA elektronische Patientenportale zur Verfügung. Dabei handelt es sich um sichere Websites, über die Patienten Informationen, z.B. die Medikationsliste und Testresultate, einsehen können. Die Portale ermöglichen auch einen sicheren Austausch von Nachrichten. Die Arztberichte bleiben aber vorerst ausserhalb des Zugriffs der Patienten.

2001 treffen sich unter dem Titel Nothing About Me Without Me Gesundheitsfachleute, Patientenvertreter und Sozialwissenschaftler aus 29 Ländern und entwickeln eine Vision für den besseren Zugang zu Informationen und zur gemeinsamen Entscheidungsfindung im Gesundheitswesen [5].

2012 wird dann die erste grosse Studie zu OpenNotes veröffentlicht [6], und erste Institutionen setzen OpenNotes um. In den USA steigt von 2016 bis 2019 die Zahl der Patienten mit Zugang zu OpenNotes von 10 auf 38 Millionen [7]. In Relation zur Bevölkerung der USA von 327 Millionen sind dies 11\% aller Einwohner. OpenNotes wird in Schweden, Estland, Island, Neuseeland und Australien flächendeckend umgesetzt und steht heute in 20 Ländern zur Verfügung [8].

\section{Wissenschaftliche Evidenz zu OpenNotes}

2010 startet in den USA eine quasi-experimentelle Interventionsstudie zu OpenNotes: 105 Hausärzte fordern über 10000 Patienten auf, die ärztlichen Verlaufseinträge über sichere Onlineportale einzusehen [6,9]. Verglichen wird mit Patienten, deren Ärzte nicht mitmachen. 92-97\% aller Patienten unterstützen OpenNotes von Anfang an [6,10]. Teilnehmende Ärzte stehen zu 69-81\% hinter OpenNotes, Nichtteilnehmende zu 16$33 \%$. Die Patientenzugriffe im Onlineportal zeigen, dass mit OpenNotes der Austausch von Mitteilungen und Rückfragen zwischen den Konsultationen nicht ändern [6]. Keine der Bedenken der Ärzte sind eingetreten: Die Konsultationen dauern nicht länger, die Rückfragen nehmen nicht zu, und die Dokumentation braucht nicht mehr Zeit. Patienten sprechen OpenNotes seltener als einmal im Monat an. An der Praxis der Ärzte ändert sich nichts. 70\% der Ärzte ergänzen im Freitext, dass OpenNotes das Vertrauen der Patienten und die Kommunikation verbessert. Nun erachten auch die Ärzte es als adäquat, ihre Notizen für die Patienten zu öffnen, und alle möchten OpenNotes behalten.

82\% der über 13000 Patienten öffnen mindestens ein Dokument. $77-87 \%$ berichten, dass OpenNotes ihnen eine bessere Kontrolle über ihre Behandlung gibt. 20$42 \%$ teilen Unterlagen mit Angehörigen. 99\% möchten OpenNotes beibehalten. 60-78\% der Patienten mit Medikation sagen, dass ihre Einnahmetreue sich verbessert. OpenNotes ist für die Arztwahl entscheidend. 3 von 5 Patienten und 1 von 3 Ärzten regen an, dass Patienten die ärztlichen Verlaufseinträge mit eigenen Kommentaren ergänzen können sollten.

OpenNotes steigert die Anzahl Konsultationen nicht [11]. Die positiven Rückmeldungen der Patienten sind unabhängig davon, ob eine psychiatrische Diagnose vorliegt oder nicht [12]. Mehr als die Hälfte will über den eigenen Zugriff hinaus Angehörigen Zugriff auf ihre Unterlagen gewähren [13]. Eine Studie, welche dies umsetzt, berichtet über viele Vorteile auch für die Bezugspersonen [14]. OpenNotes verbessert insbesondere bei Patienten mit deutlich eingeschränkter Gesundheit deren Koordination, Selbstfürsorge, Engagement und Aktivität [15].

Insgesamt geben 7\% der Patienten an, dass sie wegen des Inhalts der Unterlagen Kontakt mit dem Behandlungsteam aufgenommen haben [16]. Die Hälfte davon wollte etwas erklärt bekommen, und circa ein Drittel meldete, dass in den Aufzeichnungen etwas nicht korrekt sei. Bei 37\% aller Patienten hat OpenNotes das Verhältnis zum Arzt verbessert. Und es gilt zudem: Je mehr Unterlagen jemand liest, desto positiver ist der Einfluss auf die Arzt-Patienten-Beziehung.

\section{Einblick in Unterlagen verbessert Vertrauen}

Vor Einführung von OpenNotes schätzten 26\% der Ärzte, dass die Patienten relevante Fehler finden, und 44\% meinten, dass Patienten mit dem Inhalt nicht einverstanden seien, und befürchteten daher, dass OpenNotes ihre Arbeitsabläufe beeinträchtigen werde. Ein Jahr nach der Einführung zeigt sich aber ein anderes Bild: 51\% der Ärzte sagen übereinstimmend zu den Patienten, dass Patienten, welche die Unterlagen lesen, mehr Vertrauen in ihre Ärzte haben, und 53\% geben an, dass sich die Patientenzufriedenheit verbessert.

Eine Umfrage bei erwachsenen Patienten und bei Eltern von kranken Kindern zeigt, dass OpenNotes 75\% dieser Personen hilft, den Grund für die Untersuchung bzw. Überweisung zu verstehen [17]. 55\% berichten, dass OpenNotes ihnen hilft, Termine besser wahrzunehmen, und $75 \%$ achten dank OpenNotes vermehrt darauf, Testresultate $\mathrm{zu}$ erhalten und $\mathrm{zu}$ verstehen. Interessanterweise sind diese positiven Effekte bei bildungsfernen Personen ausgeprägter. Bei 97\% der Patienten hat sich das Vertrauen in und die Zusammenarbeit mit dem Behandlungsteam 
verbessert, und die Behandlungsziele stimmen besser überein. Eine Studie bei Betreuungspersonen liefert vergleichbare Resultate [18]. Durch die erhöhte Transparenz fördert OpenNotes das Engagement von Patienten und deren Familien sowie die Patientensicherheit.

Das Department of Veterans Affairs (VA) ist für die integrierte Versorgung der 9 Millionen Veteranen der USA zuständig [19, 20]. Das VA arbeitet mit der elektronischen Dokumentation VistA; dazu gehören das Patientenportal My HealtheVet und eine Blue Button-Schnittstelle, um eine Kopie der eigenen Daten herunterzuladen. Von 2000-2010 erfolgte ein Pilotprojekt bei 7464 Patienten mit OpenNotes und der Option zur Freigabe für Angehörige [21]. 85\% geben OpenNotes als Grund für die Teilnahme am Pilotprojekt an. 14\% geben Angehörigen Zugriff. $75 \%$ erachten die ärztlichen Notizen als einfach verständlich. Fokusgruppengespräche zeigen, dass sich OpenNotes positiv auf die Kommunikation mit den Ärzten auswirkt [22]. 2013 wird My HealtheVet mit OpenNotes ergänzt. Auch die Patienten der VA wissen dank OpenNotes besser über ihre Krankheiten Bescheid und bereiten sich besser auf die Arztbesuche vor. OpenNotes verbessert das Wissen über die eigene Gesundheit und die Selbstfürsorge. Es hilft den Patienten zwischen unterschiedlichen Ansprechpartnern zu koordinieren und stärkt ihr Engagement für eine gemeinsame Entscheidungsfindung. Einige Patienten berichten über Inkonsistenzen zwischen dem Besprochenen und den Notizen [23]. In der VA bestätigen sich alle positiven Erfahrungen: OpenNotes wird von den Patienten sehr geschätzt. Die Notizen werden gut verstanden, und das zusätzliche Wissen unterstützt die Kommunikation, eine gemeinsame Entscheidungsfindung und partizipative Medizin.

Im Weiteren gibt es drei Studien zu OpenNotes in der Onkologie [24-26]. Auch diese Patienten sind sehr zufrieden und berichten, dass sie dank OpenNotes Informationen gewinnen, die sie während der Konsultation nicht erhalten, und dass sich die Kommunikation verbessert. Sie können die Arztbesuche besser vorbereiten, während der Konsultation eine aktive Rolle wahrnehmen, sich besser einbringen und die Behandlung besser koordinieren. Während des Arztbesuchs werden die Patienten häufig von neuen Informationen überwältigt. OpenNotes reduziert die Unsicherheit und Angst, erleichtert die Kontrolle über die neue Situation und die Entscheidung über weitere Behandlungsschritte. Die Patienten schätzen es, wenn sie lesen, dass der Arzt aufgeschrieben hat, was sie selbst erzählt haben und was bei der Konsultation diskutiert wurde. Einzelne
Patienten empfinden es als belastend, über die eigene Tumorerkrankung zu lesen, und man sollte daher die Option anbieten, auf den Zugriff zu verzichten.

Im Gegensatz zur breiten Erfahrung in der ambulanten Medizin ist die Datenlage zu OpenNotes im stationären Bereich sehr spärlich. Es existieren nur Pilotstudien mit weniger als 100 Patienten sowie prinzipielle Erwägungen [27-30].

Seit 2015 wird daran gearbeitet, OpenNotes zu OurNotes zu erweitern, d.h. Patienten die Möglichkeit zu geben, eigene Dokumente zu erstellen und so zu ihrer Dokumentation beizutragen. Patienten sollen z.B. ihre persönlichen Ziele und Fragen für den nächsten Arztbesuch angeben sowie eine persönliche Zwischenanamnese in Form strukturierter Daten und als Freitext erfassen können [31, 32].

\section{Kommentar und Schlussfolgerungen}

Die verschiedenen Studien und die Berichte aus der praktischen Umsetzung zeigen, dass OpenNotes einen grossen Nutzen hat, keine relevanten Risiken bestehen und dass die Offenlegung der Notizen viel zu einer patientenzentrierten, partizipativen Medizin beiträgt. Man könnte zu bedenken geben, dass alle verfügbaren Endpunkte «nur» auf Befragungen beruhen. So wissen wir, dass die Patienten den Eindruck haben, dass sie die Medikation zuverlässiger einnehmen und sich besser um ihre Gesundheit kümmern. Noch überzeugender wäre es, wenn uns auch Daten zu harten Endpunkten wie z.B. zu aktiv gemessener adherence oder zur Anzahl Spitalaufenthalte zur Verfügung stünden. Angesichts der einstimmig positiven Resultate, der Einfachheit der Intervention und des Fehlens zusätzlicher Kosten und relevanter Risiken wären solche Daten zwar wünschenswert, sollten aber für eine Umsetzung von OpenNotes z.B. in der Schweiz nicht vorausgesetzt werden.

Eine zentrale Stärke von OpenNotes ist, dass die Bewegung von Anfang an alle Partner, d.h. Patienten, $\mathrm{Pa}$ tientenvertreter, Ärzte, Pflegfachpersonen, weitere Gesundheitsberufe, Informatik, Qualitätsmanagement und Verwaltung, einbezieht und die Erfolge institutionsübergreifend dokumentiert sind. Hilfreich ist, dass unter www.opennotes.org viele Erfahrungen und Empfehlungen für Patienten und medizinische Fachleute offen zugänglich zur Verfügung stehen und dass Institutionen, die OpenNotes umsetzen möchten, in diesen Bestrebungen grosszügig mit Know-how unterstützt werden.

Die internationalen Erfahrungen mit OpenNotes und der auch in der Schweiz bestehende Trend zu einer partizipativen Medizin legen nahe, OpenNotes in der 
Schweiz einzuführen und umzusetzen. Aufgrund der kleinräumigen Strukturen und der bisher kaum vorhandenen elektronischen Patientenportale werden bei der Umsetzung von OpenNotes verschiedene technische Fragen zu klären sein. Allenfalls könnten hier Synergien mit dem Elektronischen Patientendossier (EPD), das 2020 eingeführt werden soll, genutzt werden. Wobei zu beachten ist, dass die Einführung des EPD in der Schweiz vorerst auf die Spitäler und Pflegeheime fokussiert, die Erfahrungen mit OpenNotes aber vor allem die ambulante Medizin betreffen. Die allgemeinen Voraussetzungen für eine Einführung von OpenNotes in der Schweiz erscheinen aber aufgrund des hohen Bildungsniveaus, des breiten Zugangs zu digitalen Informationen und der partizipativen Einstellung der Bevölkerung sehr gut.

Als Fazit lässt sich sagen, dass eine partizipative Medizin und OpenNotes eng miteinander verknüpft sind. Dank OpenNotes wird sichergestellt, dass der Patient und die Angehörigen über die wesentlichen Informationen zu seiner Gesundheit verfügen, was eine grundlegende Voraussetzung für Entscheidungen auf Augenhöhe ist.

\section{Literatur}

1 Gerber M, Kraft E, Bosshard C. Shared Decision Making - Arzt und Patient entscheiden gemeinsam. Schweizerische Ärztezeitung. 2014;95:1883-89.

2 Ross SE, Lin C-T. The effects of promoting patient access to medical records: a review. J Am Med Inform Assoc 2003; 10:129-38.

3 Bundesamt für Gesundheit. Recht auf Einsicht in das Patientendossier. https://www.bag.admin.ch/bag/de/home/medizin-undforschung/patientenrechte/rechte-arzt-spital/6-recht-einsichtpatientendossier.html; accessed 12.07.2019

4 Shenkin BN, Warner DC. Giving the Patient His Medical Record: A Proposal to Improve the System. N Engl J Med 1973; 289:688-692.

5 Delbanco T, Berwick DM, Boufford JI, et al. Healthcare in a land called peoplepower: Nothing about me without me. Heal. Expect. 2001; 4:144-150.

6 Delbanco T, Walker J, Bell SK, et al. Inviting patients to read their doctors' notes: a quasi-experimental study and a look ahead. Ann Intern Med 2012; 157:461-70.

7 Opennotes Team. Opennotes: Our History: Fifty Years In the Making. https://www.opennotes.org/history/ Accessed 12.07.2019

Bitte beachten Sie zu diesem Thema auch das «Zu guter Letzt" von Yvonne Gilli auf Seite 1050 dieser Ausgabe.

\section{Korrespondenz:}

Erika Ziltener

Dachverband Schweizeri-

scher Patientenstellen

Hofwiesenstrasse 3

CH-8057 Zürich

Tel. 0443619256

dvsp[at]patientenstelle.ch

www.patientenstelle.ch
8 Esch Tobias. Achtung, Patient liest mit! Hurra, Patient liest mit! 2018. https://www.landdergesundheit.de/beteiligung/achtung-patientliest-hurra-patient-liest accessed 12.07.2019.

9 Delbanco T, Walker J, Darer JD, et al. Open notes: doctors and patients signing on. Ann Intern Med 2010; 153:121-5.

10 Walker J, Leveille SG, Ngo L, et al. Inviting patients to read their doctors' notes: Patients and doctors look ahead patient and physician surveys. Ann Intern Med 2011; 155:811-819.

11 Leveille SG, Mejilla R, Ngo L, et al. Do Patients Who Access Clinical Information on Patient Internet Portals Have More Primary Care Visits? Med Care 2016; 54:17-23.

12 Klein JW, Peacock S, Tsui JI, O’Neill SF, DesRoches CM, Elmore JG. Perceptions of Primary Care Notes by Patients With Mental Health Diagnoses. Ann Fam Med 2018; 16:343-345.
13 Jackson SL, Mejilla R, Darer JD, et al. Patients who share transparent visit notes with others: characteristics, risks, and benefits. J Med Internet Res 2014; 16:e247.

14 Wolff JL, Darer JD, Berger A, et al. Inviting patients and care partners to read doctors' notes: OpenNotes and shared access to electronic medical records. J Am Med Inform Assoc 2017; 24:e166-e172.

15 Esch T, Mejilla R, Anselmo M, Podtschaske B, Delbanco T, Walker J. Engaging patients through open notes: an evaluation using mixed methods. BMJ Open 2016; 6:e010034.

16 Bell SK, Mejilla R, Anselmo M, et al. When doctors share visit notes with patients: a study of patient and doctor perceptions of documentation errors, safety opportunities and the patient-doctor relationship. BMJ Qual Saf 2017; 26:262-270.

17 Bell SK, Folcarelli P, Fossa A, et al. Tackling Ambulatory Safety Risks Through Patient Engagement: What 10000 Patients and Families Say About Safety-Related Knowledge, Behaviors, and Attitudes After Reading Visit Notes Background: Ambulatory safety risks including delayed diagnoses or. J Patient Saf 2018. https://journals.lww.com/ journalpatientsafety/Abstract/publishahead/Tackling Ambulatory_Safety_Risks_Through_Patient.99357.aspx accessed 12.07.2019.

18 Chimowitz H, Gerard M, Fossa A, Bourgeois F, Bell SK. Empowering Informal Caregivers with Health Information: OpenNotes as a Safety Strategy. Jt Comm J Qual patient Saf 2018; 44:130-136.

19 Atkins D, Kilbourne AM, Shulkin D. Moving From Discovery to System-Wide Change: The Role of Research in a Learning Health Care System: Experience from Three Decades of Health Systems Research in the Veterans Health Administration. Annu Rev Public Health 2017; 38:467-487.

20 Nazi KM, Turvey CL, Klein DM, Hogan TP. A Decade of Veteran Voices: Examining Patient Portal Enhancements Through the Lens of User-Centered Design. J Med Internet Res 2018; 20:e10413.

21 Nazi KM, Hogan TP, McInnes DK, Woods SS, Graham G. Evaluating Patient Access to Electronic Health Records: Results From a Survey of Veterans. Med Care 2013; 51:52-56.

22 Woods SS, Schwartz E, Tuepker A, et al. Patient Experiences With Full Electronic Access to Health Records and Clinical Notes Through the My HealtheVet Personal Health Record Pilot: Qualitative Study. J Med Internet Res 2013; 15:e65.

23 Nazi KM, Turvey CL, Klein DM, Hogan TP, Woods SS. VA OpenNotes: exploring the experiences of early patient adopters with access to clinical notes. J Am Med Inform Assoc 2015; 22:380-9.

24 Pai HH, Lau F, Barnett J, Jones S. Meeting the health information needs of prostate cancer patients using personal health records. Curr Oncol 2013; 20:e561-9.

25 Kayastha N, Pollak KI, LeBlanc TW. Open Oncology Notes: A Qualitative Study of Oncology Patients' Experiences Reading Their Cancer Care Notes. J Oncol Pract 2018; 14:e251-e258.

26 Alpert JM, Morris BB, Thomson MD, Matin K, Brown RF. Implications of Patient Portal Transparency in Oncology: Qualitative Interview Study on the Experiences of Patients, Oncologists, and Medical Informaticists. JMIR cancer 2018; 4:e5.

27 Prey JE, Restaino S, Vawdrey DK. Providing hospital patients with access to their medical records. AMIA Annu Symp Proc. 2014:188493.

28 Weinert C. Giving Doctors' Daily Progress Notes to Hospitalized Patients and Families to Improve Patient Experience. Am J Med Qual 2017; 32:58-65.

29 Walker D, Menser T, Yen P-Y, McAlearney A. Optimizing the User Experience: Identifying Opportunities to Improve Use of an Inpatient Portal. Appl Clin Inform 2018; 09:105-113.

30 Yen P-Y, Walker DM, Smith JMG, Zhou MP, Menser TL, McAlearney AS. Usability evaluation of a commercial inpatient portal. Int J Med Inform 2018; 110:10-18.

31 Mafi JN, Gerard M, Chimowitz H, Anselmo M, Delbanco T, Walker J. Patients contributing to their doctors notes: Insights from expert interviews. Ann. Intern. Med. 2018; 168:302-305.

32 Safford MM. A new chapter in patient-centered care: Sharing the medical note? Ann Intern Med 2018; 168:298. 